\title{
Application and Research of SVM in Coal Mine Fan Condition Monitoring
}

\author{
Xiucai Guo, Kaixuan Wang \\ Xi' an University of Science and Technology, Xi'an, 710054, China
}

\begin{abstract}
In order to real time control of coal mine fan equipment running status, A kind of mine fan state monitoring system based on ZigBee has been designed, Through collect and processing the fan operation parameters, such as temperature, vibration, realize the function that real-time monitoring the fan's state. For mine fan condition monitoring in the process of the character of sample data is limited, support vector machine will be used to predict the trend of fan vibration signal, realize the prediction of running status of fan, provide a reference basis to Fan maintenance person. The experimental results show that support vector machine (SVM) model to predict the correctness and feasibility of fan vibration signal.
\end{abstract}

Keywords-coal mine; fan; support vector machine; state prediction.

\section{INTRODUCTION}

Fan is the important equipment of coal mine ventilation system, in the event of failure will lead to gas accumulation and explosion, endanger the safety of coal mine workers, cause huge economic losses of coal mining enterprises. In order to ensure its reliable operation, Coal put a lot of money and manpower for maintenance of the fan every year. So, effective condition monitoring is necessary for the fan. A kind of mine fan state monitoring system based on ZigBee is designed, can realize the rapid networking and the purpose of the fan running status real-time monitoring. As support vector machine adopt a new type of learning mechanism, realizes the structural risk minimization principle, has good generalization ability for the future sample, even in a long range forecast still can ensure the accuracy of fairly. Then, the support vector machine (SVM) was used for trend prediction of fan vibration signal, predict the running status of the fan in a timely manner.

\section{SYSTEM ARCHITECTURE}

Fan condition monitoring system for coal mine application, the overall system design will be as follows: data collection, analysis of the monitoring and control system communication layer. Fan condition monitoring system of coal mine based on the ZigBee, overall architecture is shown in figure 1.Data collection layer by the number of distribution on fan equipment terminal nodes to bearing of the front of the fan is temperature, bearing the front-end vibration, bearing the back-end temperature, bearing after the period of vibration, such as data acquisition, form a wireless sensor network. And sends the data to the coordinator node, complete data collection and send.
System communication layer refers to the communication between ZigBee wireless sensor network and the coordinator node and monitoring host communications, wireless sensor network communication is the communication between the terminal node and the coordinator node. Monitoring host by the coordinator node with Ethernet communication, mutual communication functions performed using master-slave response mode. Monitoring management analysis layer is monitoring software to gather up data processing, storage, the chart shows, print, control and give warning alarm functions, early warning and alarm function is based on support vector machine predictive results of the fan's vibration signal [1].

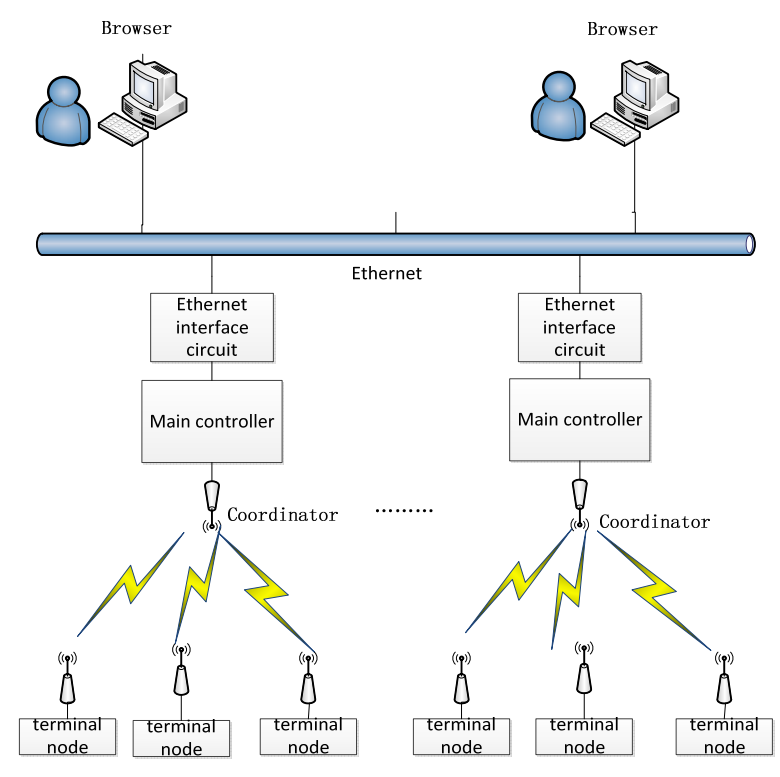

Figure 1 . The system overall architecture

\section{INTRODUCTION OF THE NONLINEAR SUPPORT VECTOR REGRESSION MACHINE}

Support vector machine (SVM) is used for classification and support vector regression machine can be used to make predictions. Due to the vibration of the fan data belongs to nonlinear signal, for linear support vector regression machine can't solve the problem of regression, the need to map sample space from a low dimensional space to high dimension space, and then find the solution with linear regression method in high dimensional space, the process is 
done by kernel function. Rather the original training set $\mathrm{U}$ introduce from European space Rn to the Hilbert space transformation. According to the Mercer theorem, kernel function is[2-3]:

$$
K\left(x_{i}, x_{j}\right)=\left(\varphi\left(x_{i}\right) \varphi\left(x_{j}\right)\right)
$$

The nonlinear optimization problem is converted into:

$$
\left\{\begin{array}{l}
\min \quad \frac{1}{2}\|\omega\|^{2} \\
\text { s.t }|\langle\omega \varphi(x)\rangle| \leq \varepsilon, i=1,2, \cdots 1
\end{array}\right.
$$

Similar to the linear regression problem, the problem of Langrange dual problem as follows:

$$
\left\{\begin{array}{l}
\max \quad-\frac{1}{2} \sum_{i=1}^{T} \sum_{j=1}^{1}\left(\alpha_{i}^{*}-\alpha_{j}\right)\left(\alpha_{j}^{*}-\alpha_{j}\right) k\left(x_{i}, x_{j}\right)+\sum_{i=1}^{t} y_{i}\left(\alpha_{i}^{*}-\alpha_{i}\right)-\varepsilon \sum_{i=1}^{t}\left(\alpha_{i}^{*}+\alpha_{i}\right) \\
0 \leq \alpha_{i}, \alpha_{i}^{*} \leq C, i=1,2, \cdots, 1 \\
\text { s.t } \sum_{i=1}^{T}\left(\alpha_{i}-\alpha_{i}^{*}\right)=0
\end{array}\right.
$$

For nonlinear regression problem must to soften hyperplane, that is relaxing constraints, within a certain range allowed to have not satisfy the constraint conditions training points exist. Therefore, introduction of slack variables $\xi_{i}$ and $\xi_{i}^{*}$ to reduce binding, including:

$$
\xi_{i}, \xi_{i}^{*} \geq 0, i=1,2, \cdots 1
$$

The optimization equation is:

$$
\left\{\begin{array}{l}
\min \frac{1}{2}\|\omega\|^{2}+c \sum_{i=1}^{l}\left(\xi_{i}+\xi_{i}^{*}\right) \\
\left\langle\omega \cdot \varphi\left(x_{i}\right)\right\rangle+b-y_{i} \leq \xi_{i}^{*}+\varepsilon, i=1,2, \cdots, 1 \\
\text { s.t } \quad y_{i}-\left\langle\omega \cdot \varphi\left(x_{i}\right)\right\rangle+b-y_{i} \leq \xi_{i}^{*}+\varepsilon, i=1,2, \cdots, l \\
\xi_{i}, \xi_{i}^{*} \geq 0, i=1,2, \cdots 1
\end{array}\right.
$$

$\mathrm{C}$ is punish coefficient, a positive number. The greater the value of $\mathrm{C}$, the greater the penalties to these doesn't meet the constraint conditions of sample points.

To solving the convex quadratic programming problem, introduce the Langrange function:

$$
\begin{gathered}
L\left(\omega, b, \alpha, \alpha^{*}\right)=\frac{1}{2}\|\omega\|^{2}+c \sum_{i=1}^{l}\left(\xi_{i}+\xi_{i}^{*}\right)-\sum_{i=1}^{I} \alpha_{i}\left(\xi_{i}+\varepsilon-y_{i}+\langle\omega \cdot x\rangle+b\right)- \\
\sum_{i=1}^{l} \alpha_{i}^{*}\left(\xi_{i}+\varepsilon-y_{i}+\langle\omega \cdot x\rangle+b\right)-\sum_{i=1}^{n} \eta\left(\xi_{i}+\xi_{i}^{*}\right)
\end{gathered}
$$$$
\alpha_{i} \quad \text { and } \quad \alpha_{i}^{*} \text { are Langrange }
$$

multiplier, $\alpha_{i}, \alpha_{i}^{*} \geq 0, i=1,2, \cdots 1$,is Positive real Number. We said $\alpha_{i}, \alpha_{i}^{*}$, which are not zero at the same time, Corresponding $\left\{\mathrm{x}_{\mathrm{t}}\right\}$ is support vector, among them $0<\alpha_{i}<C \quad \alpha_{i}^{*}=0$.

Langrange function's extremum meet requirements:

$$
\left\{\begin{array}{l}
\frac{\partial L}{\partial \omega}=0, \frac{\partial L}{\partial b}=0 \\
\frac{\partial L}{\partial \xi_{i}}=0, \frac{\partial L}{\partial \xi_{i}^{*}}=0
\end{array}\right.
$$

Solve the available:

$$
\left\{\begin{array}{l}
\omega=\sum_{i=1}^{l}\left(\alpha_{i}-\alpha_{i}^{*}\right) \varphi\left(x_{i}\right) \\
\sum_{i=1}^{1}\left(\alpha_{i}-\alpha_{i}^{*}\right)=0 \\
C-\alpha_{i}-\eta_{i}=0, i=1,2, \cdots, l \\
C-\alpha_{i}^{*}-\eta_{i}^{*}=0, i=1,2, \cdots, l
\end{array}\right.
$$

The dual problem of Langrange function is:

$$
\left\{\begin{array}{l}
\min -\frac{1}{2} \sum_{i=1}^{I} \sum_{j=1}^{I}\left(\alpha_{i}^{*}-\alpha_{j}\right)\left(\alpha_{j}^{*}-\alpha_{j}\right) k\left(x_{i}, X_{j}\right)-\sum_{i=1}^{l} y_{i}\left(\alpha_{i}^{*}-\alpha_{i}\right)+\varepsilon \sum_{i=1}^{I}\left(\alpha_{i}^{*}+\alpha_{i}\right) \\
0 \leq \alpha_{i}, \alpha_{i}^{*} \leq C, i=1,2, \cdots, 1 \\
\text { s.t } \sum_{i=1}^{I}\left(\alpha_{i}-\alpha_{i}^{*}\right)=0
\end{array}\right.
$$

Get the optimal value of $a_{i}^{*}$, and then get the w and b's value, nonlinear decision function of support vector regression machine are as follows:

$$
f(x)=\sum_{i=1}^{l}\left(\alpha_{i}-\alpha_{i}^{*}\right) K\left(x_{i}, x\right)+b
$$

When the kernel function selection radial basis kernel function, the decision function of support vector regression machine shown in the following type:

$$
f(x)=\sum_{i=1}^{1}\left(\alpha_{i}-\alpha_{i}^{*}\right) \cdot \exp \left\{\frac{-\left\|x_{i}-x\right\|^{2}}{2 \sigma^{2}}\right\}+b
$$

\section{BUILDING THE SUPPORT VECTOR REGRESSION} MACHINE PREDICTION MODEL

In this paper, using statistical methods to study the historical time series data, and the nonlinear mapping approximation data generated by learning the implicit nonlinear mechanism, and then use the mapping for time series prediction. Known a time series ${ }^{X_{t}}$ which length is $\mathrm{N}$, where ${ }^{t}=1,2, \cdots, n$. Under certain conditions the $\mathrm{m}$ to meet certain conditions, there is a smooth mapping, make

$$
x_{t}=f\left(x_{t-m}, x_{t-m+1}, \cdots, x_{t-1}\right)
$$

Come into existence. Where $\mathrm{m}$ is called the embedding dimension. Time series prediction problem is to use $\mathrm{N}-\mathrm{m}_{x_{t}}=f\left(x_{t-m}, x_{t-m+1}, \cdots, x_{t-1}\right)$ and $Y_{t}=x_{t}$ points in $\mathrm{Rm}$ form sample pair $\left(X_{i}, Y_{i}\right)(i=m+1, m+2, \cdots, N)$, Mapping $\mathrm{f}$ is estimated based on the these samples, thus $\mathrm{XN}+1$ approximation is given.

To a sampling point of fan. Until the deadline $\mathrm{t}, \mathrm{N}$ 
consecutive sequence of time vibration value in order to $\left\{x_{t}\right\}$, among $x_{t}=x(t), t=1,2, \cdots, N$. for the return of the order of time sequence $\left\{x_{t}\right\}$ vibration model is[4]:

$$
x_{t}=f\left(x_{t-m}, x_{t-m+1}, \cdots, x_{t-1}\right)
$$

The embedding dimension $\mathrm{m}$ value is called time series. According to this model can be made of the sequence $\left\{x_{t}\right\}$ vibration training sample sets (X, Y), Which input samples for continuous sample consisting of $\mathrm{M}$ order vibration value problem, For a total of $\mathrm{n}$ the vibration of the sequence $\left\{x_{t}\right\}$ in the composition of the input sample set $\mathrm{X}$ capacity of $n-m$. The output capacity of sample set for the same $\mathrm{n}-\mathrm{m}$. The input sample set $\mathrm{X}$ and output sample set $\mathrm{Y}$ as shown below.

$$
\begin{gathered}
X=\left|\begin{array}{cccc}
X_{1} & x_{2} & \cdots & X_{n-1} \\
X_{2} & X_{3} & \cdots & X_{n-m+1} \\
\cdots & \cdots & \cdots & \cdots \\
X_{m} & X_{m+1} & \cdots & X_{n-1}
\end{array}\right|_{m \times(n-m)} \\
Y=\left|X_{m+1} \quad X_{m+2} \cdots X_{n}\right|_{1 \times(n-m)}
\end{gathered}
$$

Combination type(1) the SVR based on RBF dynamic prediction model for vibration sequence $\left\{x_{t}\right\}$ is:

$$
y_{t}=\sum_{i=1}^{n-m}\left(\alpha_{i}-\alpha_{i}^{*}\right) \cdot \exp \left\{\frac{-\left\|x_{i}-x\right\|^{2}}{2 \sigma^{2}}\right\}+b
$$

\section{V.FORECAST EVALUATION INDEX}

Using the Root Mean Square Error (Root Mean Square Error, RMSE) for nonlinear approximation ability of support vector regression machine model to evaluate and forecast effect.

$$
R M S E=\sqrt{\frac{1}{N} \sum_{i=1}^{N}\left(x_{i}-y_{i}\right)^{2}}
$$

$\mathrm{N}$ is the size of the prediction set, $X_{i}$ as the observed value, $y_{i}$ is predicted.

\section{SIMULATION RESULTS}

Do the experiments of the Lorenz nonlinear signal. In the experiments, using Lorenz signal before 320 data points is used to forecast model of training, take then verifies the predicted results of 180 data points and contrast. Figure 2 for the prediction of single step of the SVM algorithm, solid line as the original data, the dotted step ahead of schedule for the SVM forecasting data. Can be seen from the figure 2, the algorithm used in the prediction error is small, the RMSE $=0.0366$.

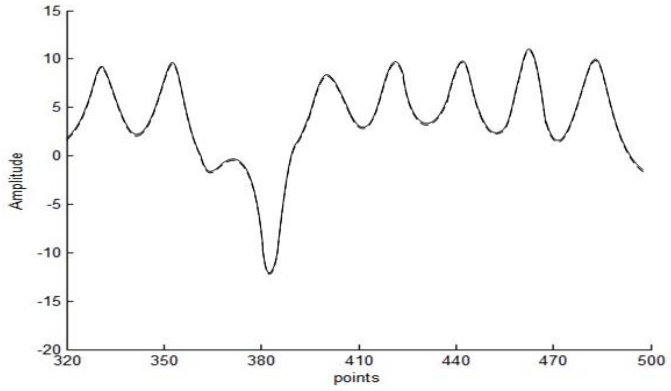

Figure 2. The prediction results of Lorenz signal

The Lorenz signal multi-step prediction experiment using the SVM algorithm, the input signal and single-step prediction experiments the input signal. Take the Lorenz signal's first 430 experimental data points, used to training, a prediction model with subsequent 70 data points to verify the correctness of the prediction results.

Figure 3, figure 4, solid line as raw data, a dotted line to the SVM forecasting data. Can be seen from the two pictures, using SVM method forecasting results in 70 forecast range and the degree of fitting of real data is better, and the predicted results of the root mean square error is 0.7926;Within the scope of the 100 steps prediction, forecasting curve can reflect the change trend of the real curve.

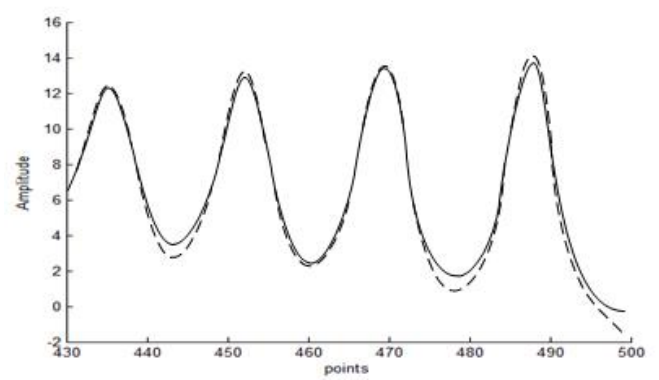

Figure 3. Result of prediction (step70)

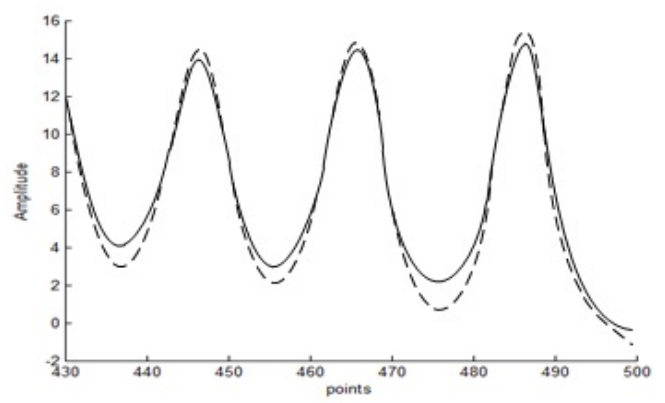

Figure 4. Result of prediction (step 100)

\section{CONCLUSION}

Aim at fan condition monitoring of coal mine, the author of this paper designed a kind of mine fan state monitoring system based on ZigBee, realize the coal mine fan equipment running status in real time and automatic 
monitoring function. Using the support vector regression machine to collected vibration signals are trend to predict these events and thus to the operation of the equipment of the fan make a prediction and warning information. The experimental results show that support vector machine in the vibration signals of the fan equipment trend prediction can meet the need, in the trend prediction of vibration signal has a good application prospect.

\section{REFERENCES}

[1] Ding Qingqing, Geng Shaobo, Gu Qibin .Design of Monitoring System for Electromechanical Equipments of Coal Mine [J]. Industr $\mathrm{y}$ and $\mathrm{M}$ ine A utomation, 2011, 06: 20-22.

[2] Hu liangmou, Cao Kqiang, Xu Haojun, Dong Xinmin. Support vector machine fault diagnosis and control technology[M].Beijing: National Defence Industry Press,2011

[3] Nello Cristianini and John Shawe-Taylor. An introduction to support vector machines and other kernel-based learning methods [M]. Beijing : China machine press , 2005

[4] LIU Lin-gang LI Xue-ren CHEN Yong-gang WU Li-xun. Aeroengine Vibration Forecasting based on Support Vector Machine [J]. Microcomputer Information, 2008, 16:289-291. 